\title{
Cytogenetics Findings in a Histiocytic Sarcoma Case
}

\author{
J. M. Alonso-Dominguez, ${ }^{1}$ M. Calbacho, ${ }^{1}$ M. Talavera, ${ }^{2}$ \\ C. Villalon, ${ }^{2}$ L. Abalo, ${ }^{1}$ J. V. Garcia-Gutierrez, ${ }^{1}$ S. Lozano, ${ }^{1}$ M. Tenorio, ${ }^{1}$ \\ J. Villarrubia, ${ }^{1}$ J. Lopez-Jimenez, ${ }^{1}$ and M. T. Ferro ${ }^{2}$ \\ ${ }^{1}$ Servicio de Hematología, Hospital Ramón y Cajal, Carretera de Colmenar Km 9, 10028049 Madrid, Spain
${ }^{2}$ Servicio de Genética, Hospital Ramón y Cajal, Carretera Colmenar KM 9, 10028034 Madrid, Spain
}

Correspondence should be addressed to J. M. Alonso-Dominguez,juanmad_es@yahoo.es

Received 29 December 2011; Accepted 19 February 2012

Academic Editors: R. Herrmann, K. Nakase, and S. D. Wagner

Copyright (C) 2012 J. M. Alonso-Dominguez et al. This is an open access article distributed under the Creative Commons Attribution License, which permits unrestricted use, distribution, and reproduction in any medium, provided the original work is properly cited.

\begin{abstract}
Histiocytic sarcoma (HS) is a neoplasm derived from histiocytes. Its diagnosis was not clear until its immunohistochemistry profile was correctly established. Not much is known about its genetic properties. We report a case of a 48 -year-old male patient whose bone marrow was almost completely occupied by monomorphic medium size neoplastic cellularity. Its immunohistochemical profile was $\mathrm{CD}^{+} 8^{+}, \mathrm{CD}^{+}, \mathrm{CD} 45^{+}$with negativity of other dendritic cells, and other lineage markers. Cytogenetic study showed 4 related clones: one with trisomy 8 and extra material on the short arms of chromosome 4; a second line with tetrasomy of chromosome 8 , add $(4)(\mathrm{p} 16)$; the third clone had the same alterations as the previous and deletion of chromosome 3 at q11; the fourth line had tetrasomy 8 and translocation $\mathrm{t}(3 ; 5)(\mathrm{q} 25 ; \mathrm{q} 35)$. To our knowledge this is the first HS case showing chromosome 8 trisomy and tetrasomy and the other described alterations.
\end{abstract}

\section{Introduction}

Histiocytic sarcoma (HS) is a neoplasm derived from histiocytes, also called macrophages. These cells are derived from bone marrow monocytes that migrate from peripheral blood to different tissues, although local proliferation also exists [1]. Therefore, it is a myeloid-derived neoplasm.

Its diagnosis was not clear until its immunohistochemical profile was correctly established. There is expression of at least one histiocytic marker: CD 68, CD 163, or lysozyme with negativity of Langerhans cell (CD1a, langerin) and follicular dendritic cell (CD21, CD35) markers. In addition CD4, CD45, CD45RO, and HLA-DR are normally positive. Epithelial membrane, melanoma, B-cell, T-cell, and myeloid markers are also negative [2-4]. There are few bona fide cases reported given that many of the neoplasms thought to be HS were T-lineage-associated hematolymphoid neoplasms [5]. Some cases occur in patients with mediastinal germ cell tumours due to the presence in these tumours of multipotential cells capable of giving rise to colonies containing macrophages and other cell types [6]. Histiocytic sarcomas usually lack clonal immunoglobulin heavy chain (IGH) or T-cell receptor (TCR) gene rearrangement, although some cases have been reported that show these clonality features [7-9].

Little is known about the cytogenetic features of this neoplasm. A case reported showed a clonal cytogenetic abnormality including $\mathrm{t}(14 ; 18)$ which was confirmed by fluorescence in situ hybridization. It also had clonal IGH gene rearrangement [9]. Other authors described a HS derived from a chronic myelomonocytic leukemia (CMMoL). HS cells were all tetraploid and had octasomy to decasomy 8 and the primitive CMMoL cells were hyperdiploid with an extra chromosome 8 [10]. We report a HS case with multiple gains of chromosome 8 and other additional abnormalities.

\section{Case Report}

A 48-year-old male patient, without interesting medical records but a congenital pendular nystagmus, was admitted 


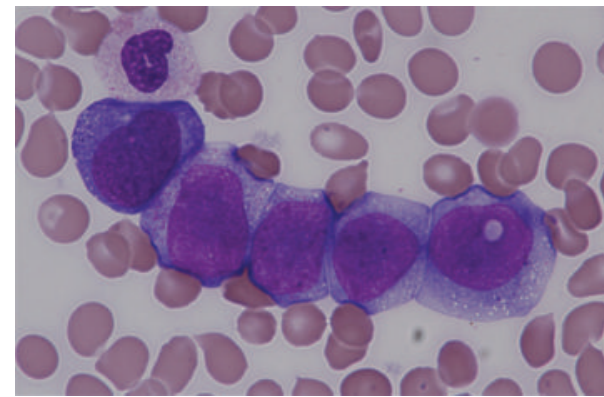

Figure 1: Peripheral blood slide review.

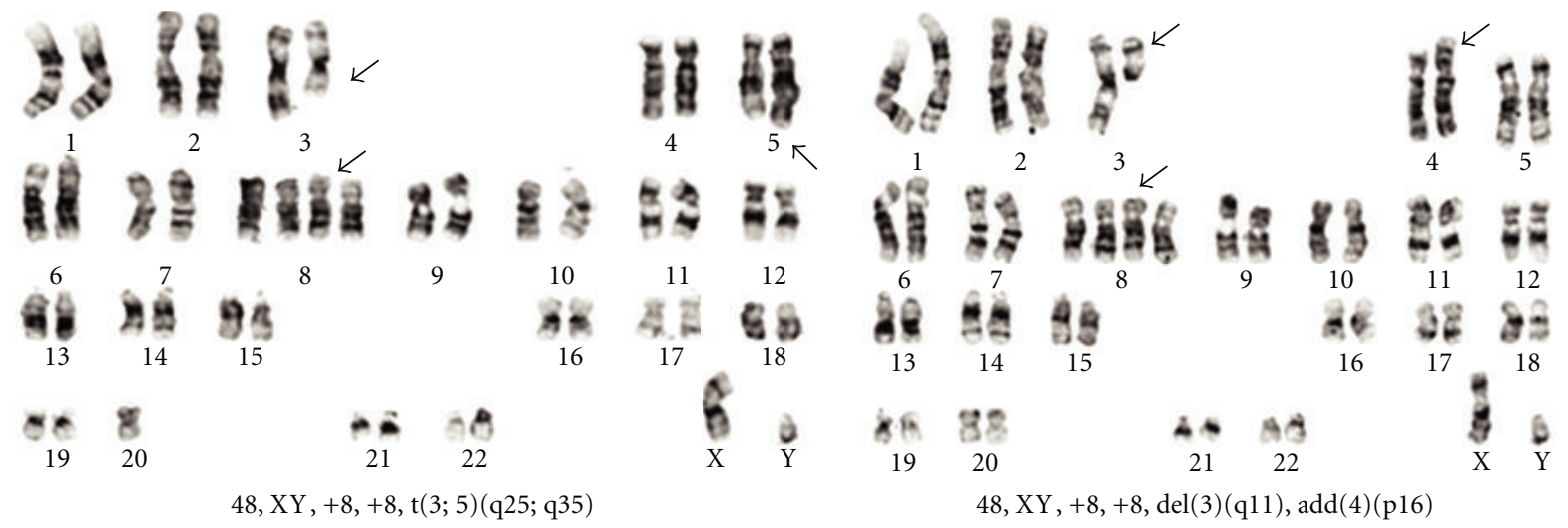

(a)

(b)

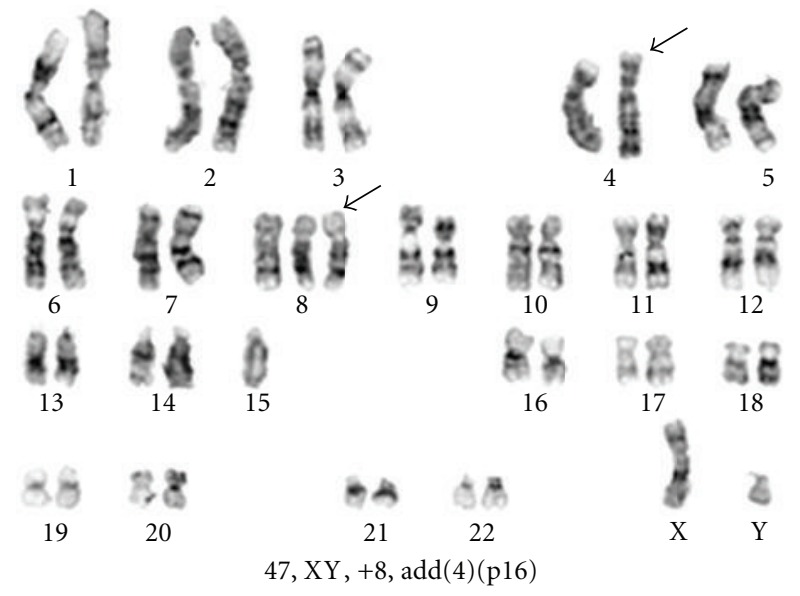

(c)

Figure 2: Histiocytic sarcoma karyotype. Alterations not pointed by arrows were not clonal.

to hospital with pancytopenia and pain in right shoulder and pelvis. On a slide review, 4\% of blasts (percentage referred to total nucleated cells) were observed and $12 \%$ of erythroblasts. The blastic cells were monomorphic, medium size, with nuclei of variable shape, round or split, with nucleoli, and presence of vacuoles, often large (Figure 1).

A bone marrow biopsy was carried out. Bone marrow was occupied almost completely by the same cells found in peripheral blood. Immunohistochemically CD68, CD4 and CD45 were positive and dendritic cells markers (CD1a, S100 and CD23) were negative excluding neoplasm derived from these related cells. Epithelial (CKAE1/AE3, CAM52), melanoma (HMB45), lymphoid (CD20, CD3, CD30, TdT), myeloid (MPO), and blast markers (CD34) were also negative.

Flow cytometry identified $42 \%$ of cells with the following profile: CD33+, CD45+, CD14-, CD11b-, CD34-, CD64+, $\mathrm{DR}+, \mathrm{CD} 4+, \mathrm{CD} 56+, \mathrm{CD} 13 \pm$.

Body Scan computed tomography (CT) showed splenomegaly of $16.5 \mathrm{~cm}$ and adenopathies of $2.5 \mathrm{~cm}$ in hepatic hilus and celiac trunk. A hypodensity surrounding the portal radicle that was attributed to extramedullar haematopoiesis 
was also detected. This could explain the splenomegaly observed.

The patient was treated with $\mathrm{CHOP} \times 4$. In the haematological cytology of the bone marrow carried out following the third chemotherapy cycle, blasts had decreased to $40 \%$ of the total cellularity.

One month after the last cycle, he was readmitted due to a paralysis of VI cranial nerve and paraesthesia in the jaw region. Minimal occupancy of sphenoidal sinus was observed in CT. Three lumbar punctures were negative for neoplastic cell. Cytarabine, methotrexate and dexamethasone were injected with each puncture. In the bone marrow aspirate the percentage of blasts rose to $99 \%$.

Cytogenetic study was performed on bone marrow 24 hours culture and showed 4 related clones: one with trisomy 8 and extra material on the short arms of chromosome 4; a second line with tetrasomy of chromosome 8 , add(4)(p16); the third clone had the same alterations as the previous and deletion of chromosome 3 at q11 and the fourth line had tetrasomy 8 and translocation $\mathrm{t}(3 ; 5)(\mathrm{q} 25 ; \mathrm{q} 35)$, similar to that described in myeloid disorders [11] (Figure 2).

The karyotype was:

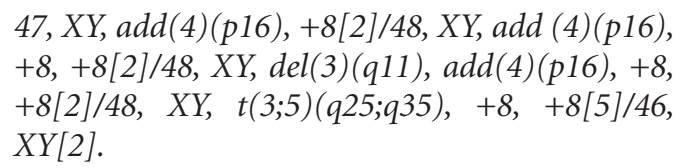

Nonclonal abnormalities were found. One of them with pentasomy 8 and other with $\operatorname{del}(7)(\mathrm{p} 11)$ had also a tetrasomy $8, \operatorname{del}(3)(\mathrm{q} 11)$, and $\operatorname{add}(4)(\mathrm{p} 16)$. This last cell might be derived from the third clone described.

With FISH analysis, there were disomic, trisomic, and tetrasomic cells for chromosome 8. The FISH study using p53, MLL, and IgH probes were also normal.

Two other different chemotherapy cycles containing ifosfamide, carboplatin plus etoposide, and methotrexate plus cytarabine were administered. The patient died of progressive disease 6 months after diagnosis.

\section{Discussion}

Our case was studied by using different techniques and its immunohistochemical pattern was conclusive in order to establish the correct diagnosis following current diagnosis criteria [4].

To our knowledge this is the first HS case showing chromosome 8 trisomy and tetrasomy and the other described alterations. The most likely evolution of the neoplasm was from an initial trisomy 8 clone that gained an extra chromosome 8 copy and developed the additional alterations.

Trisomy 8 is one of the most common chromosomal abnormalities in myeloid malignancies occurring in approximately $10-20 \%$ of cytogenetically abnormal acute myeloid leukemias (AML), myelodysplastic syndromes (MDSs), and myeloproliferative disorders (MPD) [12-14].

Chromosome 8 gains, from trisomies to pentasomies, have been described in a series of myeloid malignancies corresponding predominantly to neoplasm with monocytic differentiation (AML-M4, AML-M5, and CMMoL) [14-20].
Taking this into consideration, our cytogenetic findings in this HS case support its myelomonocytic origin and shed light on genetic alterations underlying this uncommon neoplasm.

HS is a very aggressive tumour according to the few series published so far $[3,21]$. The extension of the disease and the size seem to be the most important prognostic factors. Our case had bone marrow involvement. This advanced stage and the complex karyotype could explain the severity of this case.

\section{References}

[1] G. J. Randolph, V. Angeli, and M. A. Swartz, "Dendritic-cell trafficking to lymph nodes through lymphatic vessels," Nature Reviews Immunology, vol. 5, no. 8, pp. 617-628, 2005.

[2] S. A. Pileri, T. M. Grogan, N. L. Harris et al., "Tumours of histiocytes and accessory dendritic cells: an immunohistochemical approach to classification from the International Lymphoma Study Group based on 61 cases," Histopathology, vol. 41, no. 1, pp. 1-29, 2002.

[3] J. A. Vos, S. L. Abbondanzo, C. L. Barekman, J. W. Andriko, M. Miettinen, and N. S. Aguilera, "Histiocytic sarcoma: a study of five cases including the histiocyte marker CD163," Modern Pathology, vol. 18, no. 5, pp. 693-704, 2005.

[4] S. H. Swerdlow, E. Campo, and N. L. Harris, Eds., WHO Classification of Tumours of Haematopoietic and Lymphoid Tissues, International Agency for Research on Cancer, Lyon, France, 4th edition, 2008.

[5] M. S. Wilson, L. M. Weiss, K. C. Gatter, D. Y. Mason, R. F. Dorfman, and R. A. Warnke, "Malignant histiocytosis. A reassessment of cases previously reported in 1975 based on paraffin section immunophenotyping studies," Cancer, vol. 66, no. 3, pp. 530-536, 1990.

[6] C. A. Cudennec and G. R. Johnson, "Presence of multipotential hemopoietic cells in teratocarcinoma cultures," Journal of Embryology \& Experimental Morphology, vol. 61, pp. 51-59, 1981.

[7] A. F. Lauritzen, G. Delsol, N. E. Hansen et al., "Histiocytic sarcomas and monoblastic leukemias: a clinical, histologic, and immunophenotypical study," American Journal of Clinical Pathology, vol. 102, no. 1, pp. 45-54, 1994.

[8] W. Chen, S. K. Lau, D. Fong et al., "High frequency of clonal immunoglobulin receptor gene rearrangements in sporadic histiocytic/dendritic cell sarcomas," American Journal of Surgical Pathology, vol. 33, no. 6, pp. 863-873, 2009.

[9] E. Hayase, M. Kurosawa, M. Yonezumi, S. Suzuki, and H. Suzuki, "Aggressive sporadic histiocytic sarcoma with immunoglobulin heavy chain gene rearrangement and $\mathrm{t}(14 ; 18)$, International Journal of Hematology, vol. 92, no. 4, pp. 659663, 2010.

[10] M. Mori, A. Matsushita, Y. Takiuchi et al., "Histiocytic sarcoma and underlying chronic myelomonocytic leukemia: a proposal for the developmental classification of histiocytic sarcoma," International Journal of Hematology, vol. 92, no. 1, pp. 168-173, 2010.

[11] S. C. Raimondi, I. D. Dube, M. B. Valentine et al., "Clinicopathologic manifestations and breakpoints of the $t(3 ; 5)$ in patients with acute nonlymphocytic leukemia," Leukemia, vol. 3, no. 1, pp. 42-47, 1989.

[12] D. Grimwade, H. Walker, F. Oliver et al., "The importance of diagnostic cytogenetics on outcome in AML: analysis of 1,612 
patients entered into the MRC AML 10 trial. The Medical Research Council Adult and Children's Leukaemia Working Parties," Blood, vol. 92, no. 7, pp. 2322-2333, 1998.

[13] F. Mertens, B. Johansson, S. Heim, U. Kristoffersson, and F. Mitelman, "Karyotypic patterns in chronic myeloproliferative disorders: report on 74 cases and review of the literature," Leukemia, vol. 5, no. 3, pp. 214-220, 1991.

[14] F. Sole, B. Espinet, G. F. Sanz et al., "Incidence, characterization and prognostic significance of chromosomal abnormalities in 640 patients with primary myelodysplastic syndromes. Grupo Cooperativo Espanol de Citogenetica Hematologica," British Journal of Haematology, vol. 108, no. 2, pp. 346-356, 2000.

[15] "Morphologic, immunologic and cytogenetic (MIC) working classification of the acute myeloid leukaemias. Second MIC Cooperative Study Group," British Journal of Haematology, vol. 68, no. 4, pp. 487-494, 1988.

[16] R. Berger, G. Flandrin, A. Bernheim et al., "Cytogenetic studies on 519 consecutive de novo acute nonlymphocytic leukemias," Cancer Genetics and Cytogenetics, vol. 29, no. 1, pp. 9-21, 1987.

[17] M. T. Ferro, Y. Vázquez-Mazariego, S. Ramiro et al., "Trisomy/ tetrasomy of chromosome 8 and $+\mathrm{i}(8 \mathrm{q})$ as the sole chromosome abnormality in three adult patients with myelomonocytic leukemia," Cancer Genetics and Cytogenetics, vol. 120, no. 2, pp. 163-165, 2000.

[18] Y. Hamey, N. Dean, J. V. Catalano, and L. J. Campbell, "Pentasomy of chromosome 8 in chronic myelomonocytic leukemia," Cancer Genetics and Cytogenetics, vol. 103, no. 2, pp. 164-166, 1998.

[19] S. N. J. Sait, A. Raza, and A. A. Sandberg, "Tetrasomy of chromosome 8: an interesting and rare cytogenetic phenomenon in acute nonlymphocytic leukemia," Cancer Genetics and Cytogenetics, vol. 27, no. 2, pp. 269-271, 1987.

[20] K. Paulsson, T. Säll, T. Fioretos, F. Mitelman, and B. Johansson, "The incidence of trisomy 8 as a sole chromosomal aberration in myeloid malignancies varies in relation to gender, age, prior iatrogenic genotoxic exposure, and morphology," Cancer Genetics and Cytogenetics, vol. 130, no. 2, pp. 160-165, 2001.

[21] J. L. Hornick, E. S. Jaffe, and C. D. M. Fletcher, "Extranodal histiocytic sarcoma: clinicopathologic analysis of 14 cases of a rare epithelioid malignancy," American Journal of Surgical Pathology, vol. 28, no. 9, pp. 1133-1144, 2004. 


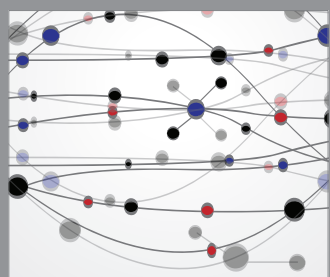

The Scientific World Journal
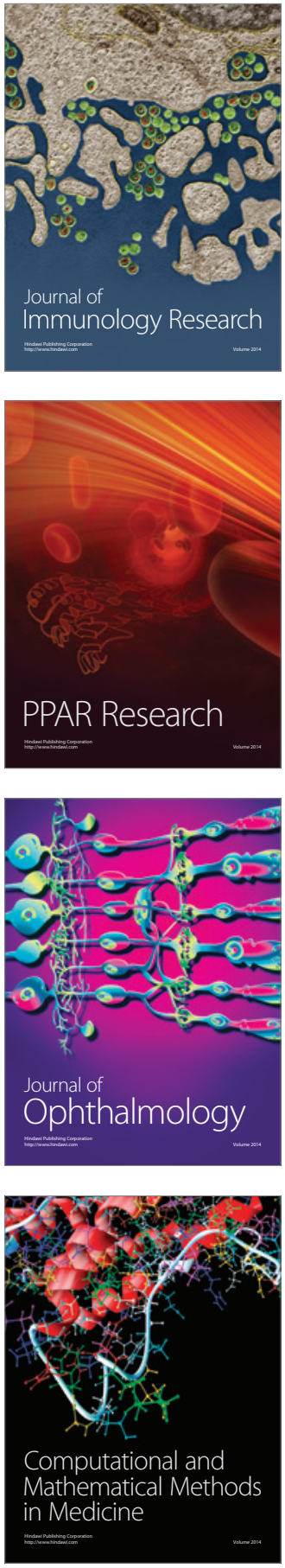

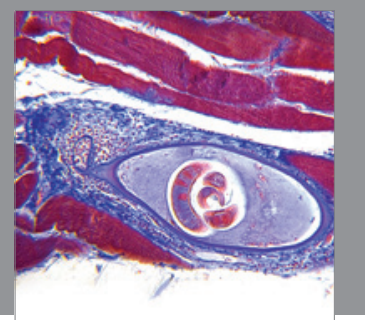

Gastroenterology

Research and Practice
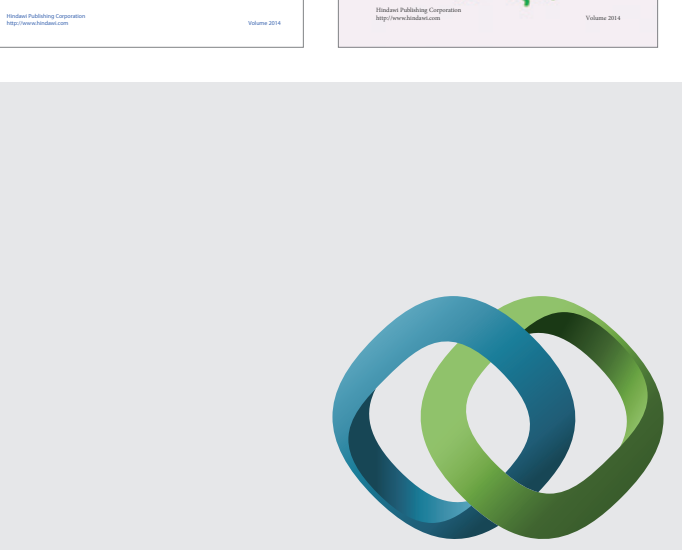

\section{Hindawi}

Submit your manuscripts at

http://www.hindawi.com
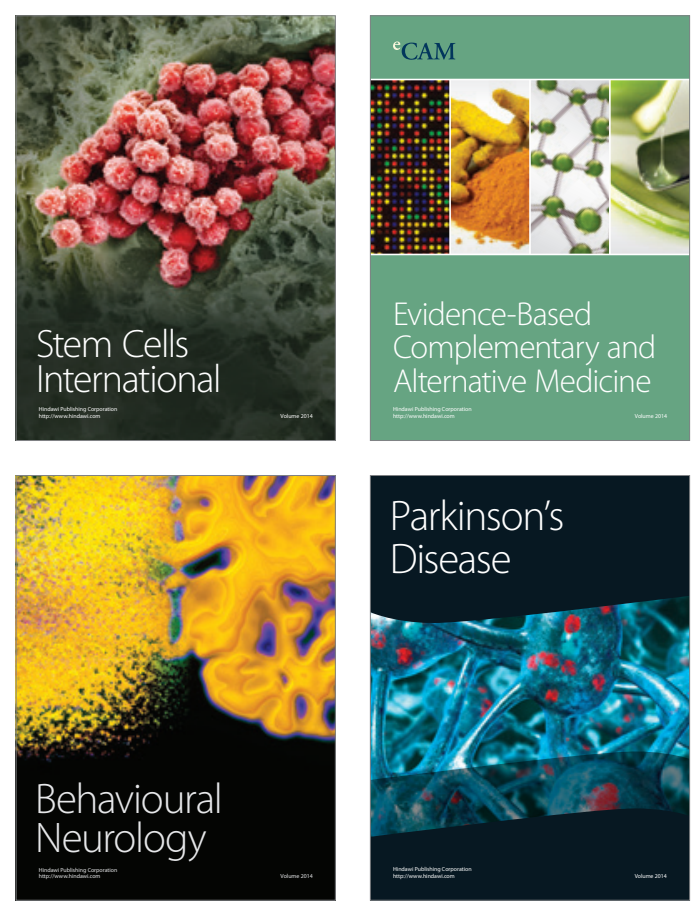

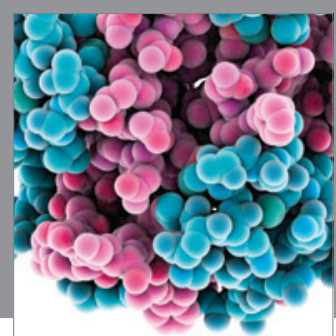

Journal of
Diabetes Research

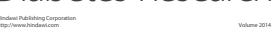

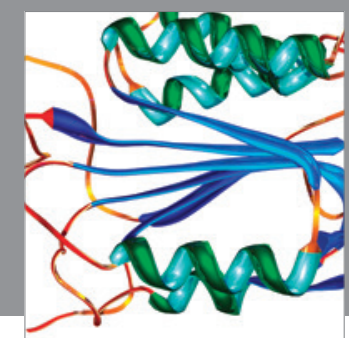

Disease Markers
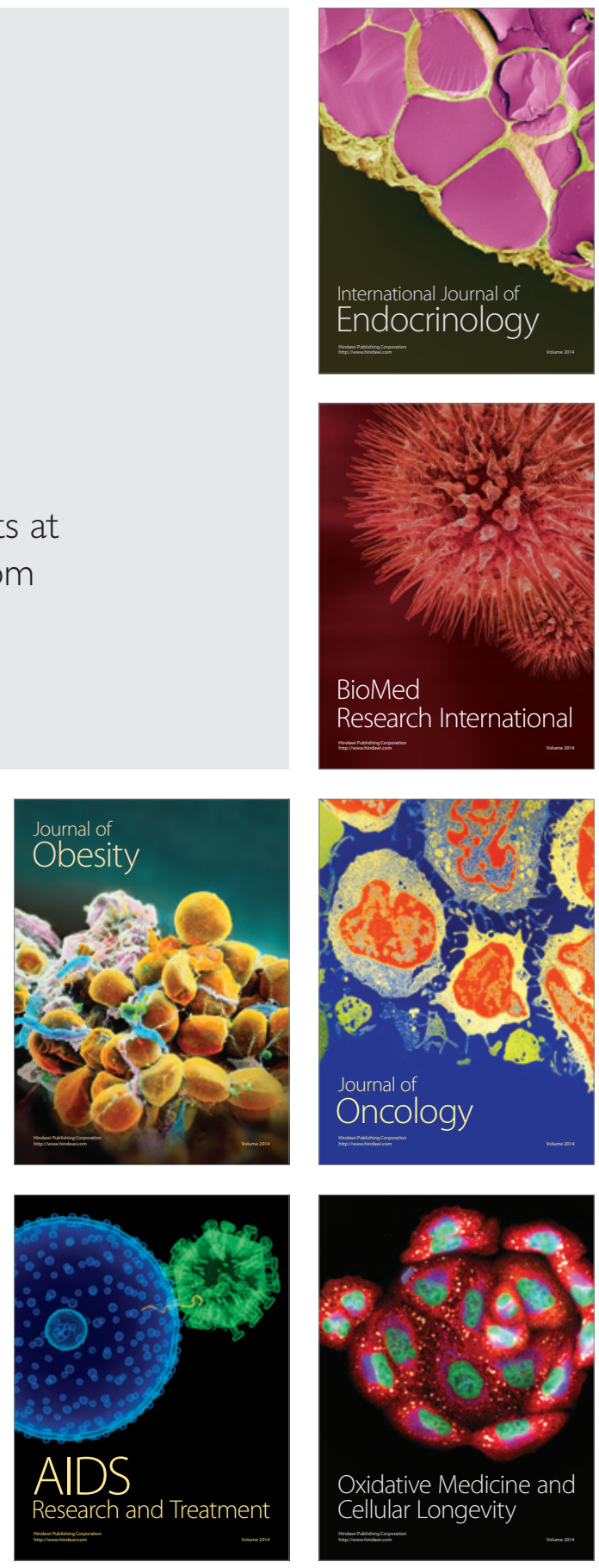